POS PROCEEDINGS

\title{
Temporal signatures of the Cherenkov light induced by extensive air showers of cosmic rays detected with the Yakutsk array
}

\author{
Anatoly Ivanov* and Lev Timofeev \\ Shafer Institute for Cosmophysical Research \& Aeronomy \\ 31 Lenin Ave., Yakutsk, 677980, Russian Federation \\ E-mail: ivanovdikfia.vsn.ru
}

We are analysing temporal characteristics of signals from the wide field-of-view (WFOV) Cherenkov telescope detecting extensive air showers (EAS) of cosmic rays (CR) in coincidence with surface detectors of the Yakutsk array. Our aim is to reveal causal relationships between measured characteristics and physical properties of EAS.

The 34th International Cosmic Ray Conference,

30 July- 6 August, 2015

The Hague, The Netherlands

\footnotetext{
* Speaker.
} 


\section{Introduction}

The data set of EAS events detected in coincidence between the wide field-of-view (WFOV) Cherenkov telescope (CT) and the surface scintillation counters of the Yakutsk array is analyzed in order to elucidate the shower development parameters in the atmosphere basing on the data from CT.

The Yakutsk array detectors are described in [ [प, D]. A description of WFOV CT can be found in []]. An example of the WFOV Cherenkov telescope output signals in a particular EAS event is given in Fig. 四.

The data are accumulated in 2012-2015 observational period where coincident EAS events are detected. A sample of showers is selected in the energy interval $\left(2 \times 10^{16}, 10^{19}\right) \mathrm{eV}$ and has a mean energy $0.3 \mathrm{EeV}\left(1 \mathrm{EeV}=10^{18} \mathrm{eV}\right)$. Additional cuts are applied to reject showers with axes out of the array area and zenith angles $\theta>60^{\circ}$. A specific cut used is for saturated signals where EAS events generate more Cherenkov light than CT can detect properly. These signals are rejected, too. A number of EAS events survived after cuts is 733 .

\section{Pulse duration as a function of the distance to the shower core}

We are focused here on the pulse shape parameters of the Cherenkov signal from EAS. The main feature of the Cherenkov signal is its duration rising with the shower core distance due to geometrical reasons. The first measurements of the parameter were made in Yakutsk [ [G] and Haverah Park [ [వ]. The most recent results are provided by the Tunka array [6]. Our measurement of the full width at half-maximum (FWHM) of the signal confirms the previous results (Fig. [2).

The energy ranges relevant are: Haverah Park $\langle E\rangle \sim 0.2 \mathrm{EeV}$; Yakutsk, $1975 E \in(0.001,1)$ EeV; Tunka $E \in(0.003,0.03) \mathrm{EeV}$.

We have analyzed FWHM dependence on other shower parameters: zenith and azimuth angles, energy. No significant variation exceeding instrumental errors is found except primary energy
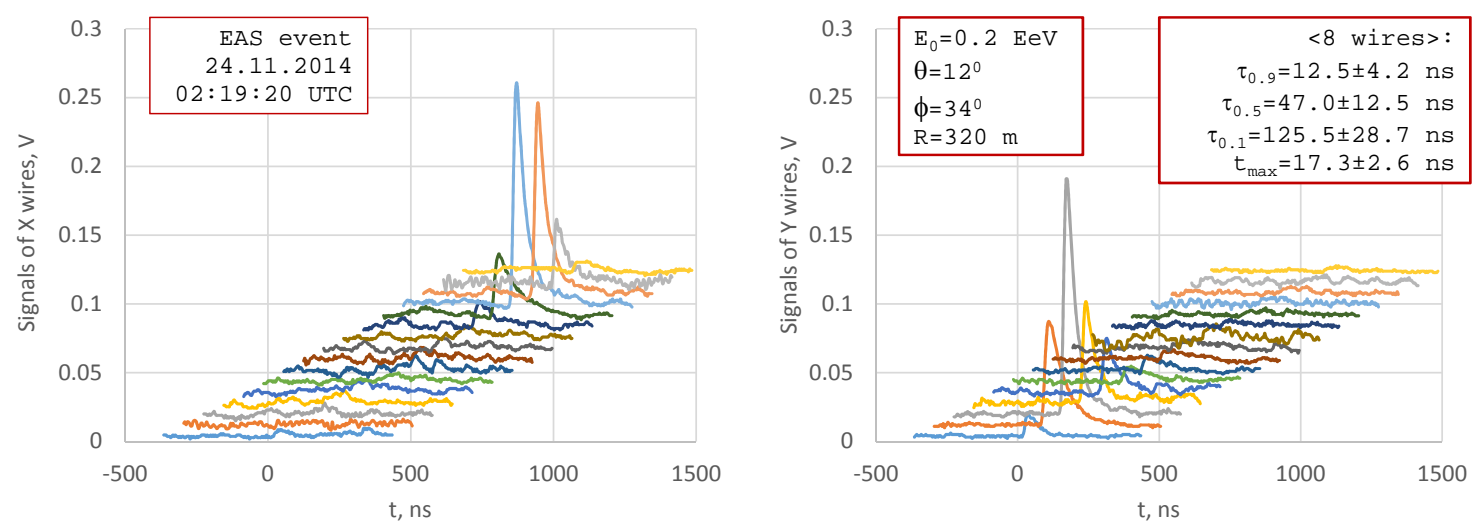

Figure 1: Signals of 32 wires from multi-anode PMT in the focus of telescope. EAS parameters in a particular shower are estimated using data of the surface detectors. Pulse durations $(\tau)$ are measured at 0.1 , $0.5,0.9$ levels of the pulse maximum $V_{\max }$. Time difference $\left(t_{\max }\right)$ between $V_{\max }$ and $0.1 V_{\max }$ of the rising signal is shown in the right panel. 


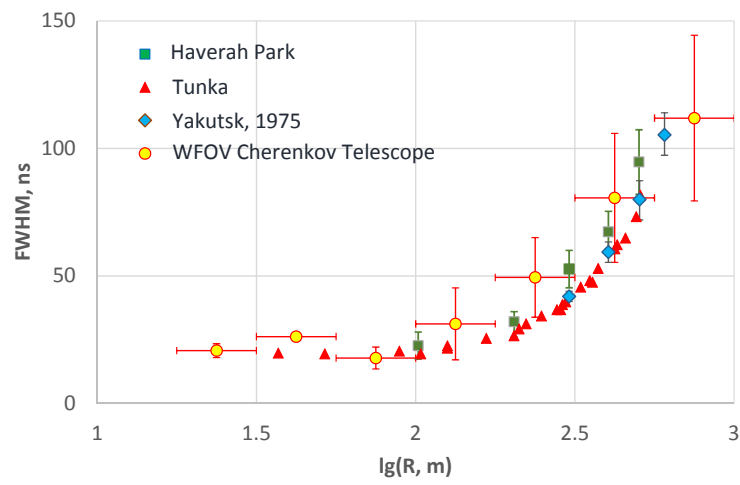

Figure 2: Full width at half-maximum of the Cherenkov signal measured by EAS arrays. HP and Yakutsk data are averaged over samples of showers, while Tunka signal is detected in a particular EAS event. Vertical bars are statistical errors while horizontal bars indicate the shower core distance, $R$, bins.

dependence. In Fig. [3 the signal width is shown as a function of energy. A systematic rise of the width is revealed in the shower core distance interval 56-100 m, although not exceeding experimental errors.

Application of $\operatorname{FWHM}(R)$ function is possible in the shower core location procedure in the array plane. The method was pioneered by John Linsley [ [U] using charged particle signal width vs $R$. While in the Yakutsk array group the particle and Cherenkov photon density distribution functions are in use to locate the shower core, $\operatorname{FWHM}(R)$ function can be used additionally (or instead) to refine the core coordinates in the array plane.

\section{A Pythagorean method to estimate the distance to the shower maximum in Cherenkov light}

Having the time delay, $\Delta t$, of the Cherenkov signal maximum in detector relative to the shower axis crossing the array plane at $R$, we are able to calculate the distance, $L_{d}$, to the point where the maximum of Cherenkov photons is emitted, $x_{\max }^{\text {Cher }}$. Other shower parameters needed are the coordinates of the shower axis and EAS arrival angles. In our case these parameters are provided by the set of synchronized surface detectors of the Yakutsk array.

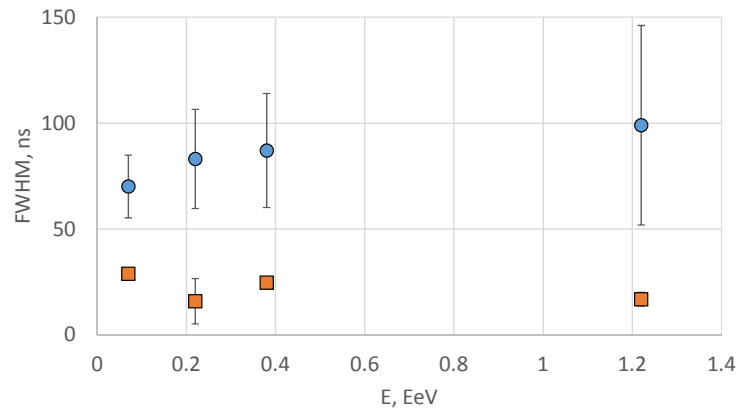

Figure 3: Energy dependence of FWHM. The signal width is measured in EAS core distance intervals $R \in(56,100) \mathrm{m}$ (squares) and $R \in(316,562) \mathrm{m}$ (circles). 


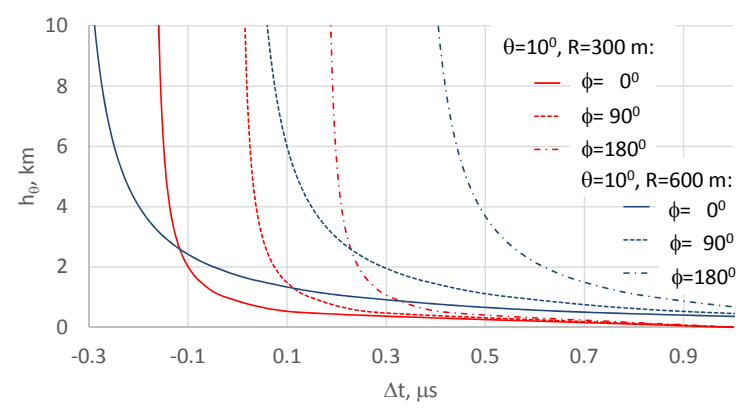

Figure 4: The height of Cherenkov light emission maximum as a function of time difference, $\Delta t$, between Cherenkov photons arriving to the detector and to the shower core in the array plane. Azimuth angle, $\phi$, is between the detector and the shower axis projection on the array plane.

The time difference is determined by triangles consisting of $R, h_{\theta}, h, L_{d}$; that is why we call the method Pythagorean:

$$
c \Delta t=\sqrt{h_{\theta}^{2}+R^{2}-2 R h_{\theta} \sin \theta \cos \phi}-h_{\theta},
$$

where $\phi, \theta$ are shower arrival angles; $h_{\theta}=h \sec \theta$ is the height of the maximum; $\mathrm{c}=0.3 \mathrm{~m} / \mathrm{ns}$. In our previous paper [园] a relation is given with slightly different notations. A solution is given by

$$
h_{\theta}=\frac{0.5\left(R^{2}-(c \Delta t)^{2}\right)}{c \Delta t+R \sin \theta \cos \phi}
$$

and is illustrated in Fig. 因.

Rather large values of the derivative $d h_{\theta} / d \Delta t$ near the time difference threshold restrict the maximum location possibilities within narrow limits in the time difference accuracy. On the other hand, relatively small heights can be resolved with reasonable time resolution.

We illustrate in Fig. [ the maximum height location accuracy achievable with the different shower core and time measurement errors. The key objective in this context is improvement of synchronization of the array detectors timing.

While the height of maximum found is determined by geometrical factors only, such as the shower core distance, zenith angle, etc., a relation to the shower maximum (in the total number of

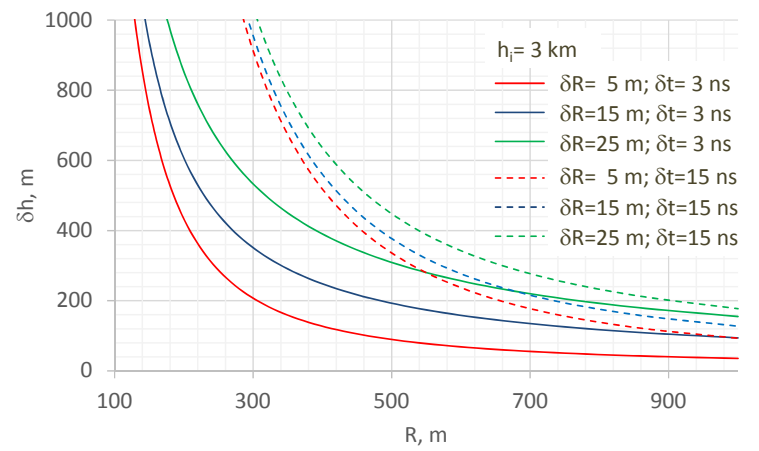

Figure 5: Estimation of the $h_{m}$ reconstruction accuracy as a function of the shower core distance. Different EAS core location and timing errors are given. 
electrons, $x_{m}^{e}$ ) position is more complicated. Unknown angular distribution of electrons, as well as the radial distribution in the shower affect $x_{m}^{C h e r}$. A model simulation of the shower development and the Cherenkov light emission is needed to reconstruct $x_{m}^{e}$ basing on the evaluation of $x_{m}^{C h e r}$.

\section{Conclusions}

Coincident detection of EAS events by the surface detectors of the Yakutsk array and WFOV Cherenkov telescope is performed during three observational seasons (winters). 733 showers are selected for analysis applying quality cuts.

A FWHM parameter of EAS is measured as a function of the shower core. We confirm the results of previous measurements within the primary energy interval $(0.02,10) \mathrm{EeV}$. The function $\mathrm{FWHM}(\mathrm{R})$ can be used to locate the shower axis in the array plane along with the particle density distribution functions.

A new approach is explored in estimation of the distance to the shower maximum basing on the time difference between Cherenkov photons arriving to the detector and the shower core in the array plane. Fast optical detectors and nanosecond-order synchronization system of the array are essential in technical realization of the method.

\section{Acknowledgments}

We are grateful to the Yakutsk array staff for the data acquisition and analysis. The work is supported by RFBR grant no. 13-02-12036.

\section{References}

[1] V.P. Egorova et al. J. Phys. Soc. Japan, B, 70 (2001) 9.

[2] A.A. Ivanov et al. NJPh, 11 (2009) 065008.

[3] A.A. Ivanov et al. NIMPA, 772 (2015) 34.

[4] N.N. Kalmykov et al., JETP Lett. 21 (1975) 66.

[5] R.T. Hammond et al., Nuovo Cim. C1, (1978) 315.

[6] V.V. Prosin et al., Nucl. Phys. B - Proc. Suppl. 190 (2009) 247.

[7] J. Linsley, Proc. 19th ICRC. La Jolla. 9 (1985) 434. 